pertinent as the number of diseases found to be associated with channel abnormalities grows. Mutations in TRPML1 cause a lysosomal storage disorder affecting children, and TPCs have been implicated in fatty liver disease, Ebola infection and several neurodegenerative disorders ${ }^{17,18}$. In this context, a human TPC structure would be most welcome.

Another challenge is to resolve the structure of TPC2. This protein is regulated by NAADP and $\mathrm{PI}(3,5) \mathrm{P}_{2}$, but not by changes in voltage begging the question of how conformational changes in one TM domain are transmitted to the other to allow channel opening. No doubt, TPCs will reveal further secrets through forthcoming structures.

Sandip Patel is in the Department of Cell and Developmental Biology, University College

London, London WC1E 6BT, UK.

e-mail:patel.s@ucl.ac.uk

1. Patel, S. Sci. Signal. 8, re7 (2015).

2. Kilpatrick, B. S. et al. Cell Rep. 18, 1636-1645 (2017).

3. She, J. et al. Nature 556, 130-134 (2018).

4. Guo, J. et al. Nature 531, 196-201 (2016).

5. Kintzer, A. F. \& Stroud, R. M. Nature 531, 258-264 (2016).

6. Brailoiu, E. et al. J. Cell Biol. 186, 201-209 (2009).

7. Calcraft, P. J. et al. Nature 459, 596-600 (2009).
8. Churchill, G. C. et al. Cell 111, 703-708 (2002)

9. Wang, X. et al. Cell 151, 372-383 (2012).

10. Rybalchenko, V. et al. J. Biol. Chem. 287, 20407-20416 (2012)

11.Cang, C., Bekele, B. \& Ren, D. Nature Chem. Biol. 10, 463-469 (2014).

12.Patel, S., Churamani, D. \& Brailoiu, E. Cell Calcium 68, 1-4 (2017)

13.Ruas, M. et al. EMBO J. 34, 1743-1758 (2015).

14. Schmiege, P., Fine, M., Blobel, G. \& Li, X. Nature 550 , 366-370 (2017).

15. Hirschi, M. et al. Nature 550, 411-414 (2017).

16.Chen, Q. et al. Nature 550, 415-418 (2017).

17.Grimm, C., Butz, E., Chen, C.-C., Wahl-Schott, C. \&

Biel, M. Cell Calcium 67, 148-155 (2017).

18.Patel, S. Messenger 5(1-2), 24-29 (2016).

This article was published online on 21 March 2018.

\title{
Bacterial persister cells tackled
}

\section{Chronic infections can be hard to treat because slow-growing bacteria known as persister cells are usually unharmed by antibiotics. The identification of molecules that target such cells might provide a solution. SEE LETTER P.103}

\section{JULIAN G. HURDLE \& ADITI DESHPANDE}

$\mathrm{T}$ he use of antibiotics to treat an infection can be unsuccessful when bacteria evade such drugs through genetic changes that endow them with antibiotic resistance. Pathogenic bacteria can also avoid antibiotic-mediated destruction through another route: some bacterial cells enter a metabolically inactive or dormant state to become persister cells, which grow slowly or not at all. Most antibiotics were discovered in experiments that tested the ability of compounds to inhibit bacterial growth, and they are therefore often ineffective for treating non-growing persister cells ${ }^{1}$. On page 103, Kim et al. ${ }^{2}$ now report the identification of small molecules that can kill persister cells.

Persister cells are the source of many of the recurrent bacterial infections that affect people, for example those associated with implanted medical devices, such as the heart infection endocarditis, and also lung infections that can arise in cystic fibrosis ${ }^{1,3}$. Curing such chronic infections can require surgery, which places an added health burden on patients.

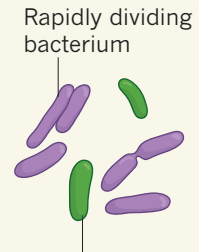

Slow-growing persister bacterium b

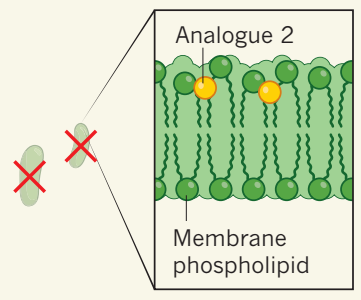

Membrane distortion causes bacterial cell death

Figure 1 | A retinoid compound destroys bacterial persister cells. a, Bacterial populations commonly consist of rapidly dividing cells and slow-growing cells that are known as persister cells. When bacteria are treated with conventional antibiotics, the rapidly dividing cells are destroyed. However, the persister cells can remain, giving rise to chronic infection. $\mathbf{b}$, Kim et al. ${ }^{2}$ report a retinoid compound called analogue 2 that was optimized for targeting persister bacteria. In a mouse model of bacterial infection, the authors found that analogue 2 could kill persister cells. Electron microscopy and computer modelling revealed that analogue 2 probably binds to phospholipid molecules in the bacterial membrane, resulting in membrane distortion that might help to kill the bacteria.

And any necessary extended periods of treatment with antibiotics will increase the probability that bacteria evolve resistance. The development of treatments for killing persister cells is therefore urgently needed ${ }^{4}$, especially to target persisters that arise in infections with strains of the 'superbug' bacterium methicillin-resistant Staphylococcus aureus (MRSA), which is resistant to several common antibiotics. Infection with MRSA is associated with illness and death, particularly among people with invasive infections ${ }^{5}$.

Kim and colleagues decided to search for molecules that could offer protection from MRSA infection, using the roundworm Caenorhabditis elegans as a model system. Taking a high-throughput approach, the authors tested the ability of around 82,000 small synthetic molecules to protect worms from death mediated by MRSA infection. Of the 185 compounds that conferred protection, the authors focused on two molecules called CD437 and CD1530, both of which kill MRSA cells rapidly and can also target Enterococcus faecium, a bacterium that is linked to endocarditis. Unfortunately, these compounds had no effect against Gram-negative bacteria a group that includes Escherichia coli - for which new therapeutic options are desperately needed because they, too, can form antibioticresistant superbugs.

CD437 and CD1530 belong to a class of molecule known as the retinoids, which are structurally similar to vitamin A. Since the 1960s, retinoids have been developed to treat various conditions, including acne ${ }^{6}$. Subsequent synthetic modification of the retinoids has therefore generated derivatives that are often present in chemical libraries used in drug discovery.

The authors concluded that prompt killing of MRSA cells occurred when the two retinoid molecules distorted the structure of the bacterial membrane's lipid bilayer. Kim and colleagues then carried out electronmicroscopy studies, which revealed that the retinoid treatment caused curvature and folding of the bacterial membrane but did not result in membrane destruction.

The bacterial membrane is a permeability 
barrier that is essential for many cellular functions ${ }^{1}$, and it contains proteins that are crucial to controlling the uptake of nutrients, the release of waste and the production of energy in the form of molecules of ATP. By distorting the membrane, the retinoid molecules probably affect the import and export of solutes, as well as several other essential cellular functions that rely on membrane integrity. However, a mode of antibacterial action that involves simply attacking the bacterial membrane is not guaranteed to kill persister cells. For example, the authors report that the membrane-targeting antibiotics nigericin and valinomycin do not kill MRSA persister cells.

Through computer simulations, the authors explored how the retinoid molecules might interact with the bacterial membrane. They determined that the polar side groups of CD437 and CD1530 could bind to the hydrophilic heads of phospholipids in the membrane, enabling the retinoid molecules to lodge in the lipid bilayer of a bacterium. Such simulations are a powerful tool that could be used to guide the optimization of antibiotics that can selectively attack the lipid bilayer of bacterial membranes without disrupting their mammalian counterparts and causing toxicity to patients.

A major concern is how to optimize small molecules such as the retinoids to enable such selectivity. Although the authors found that CD437 and CD1530 did not destroy the lipid membranes of human red blood cells, the

molecules were able to kill human liver-cancer cells grown in vitro, which is consistent with the previously reported anticancer properties of the retinoids ${ }^{6}$.

The authors generated structural variants of CD437, producing a compound they called analogue 2 that did not kill normal or cancerous human liver cells grown in vitro, but did retain the ability to kill MRSA persister cells (Fig. 1). In experiments in mice, analogue 2 remained in circulation in the animals' bodies for several hours at

"Some bacterial cells enter a metabolically inactive or dormant state to become persister cells." high enough concentrations to kill MRSA persister cells, but did not give rise to signs of toxicity such as liver or kidney damage. Remarkably, the authors showed in mice that analogue 2 could tackle what would generally be considered to be a treatment-resistant form of MRSA. This animal model mimics chronic infection with MRSA in immunocompromised people, for whom the prognosis is poor with conventional antibiotic treatments such as vancomycin because of the large number of MRSA persister cells that are present ${ }^{4,7}$.

The authors found that the effects of analogue 2 on bacterial infections could be boosted by the presence of the antibiotic gentamicin, an inhibitor of bacterial protein synthesis that lacks activity against persister cells. It will be interesting to determine whether
MRSA persister cells respond to the retinoids by reactivating their cellular metabolism, thereby making them more susceptible to being killed by drugs such as gentamicin that would otherwise be ineffective.

Molecules such as analogue 2 might be suitable candidates for drugs that decrease the time required to successfully treat chronic infections that are characterized by high loads of dormant bacteria. In an era in which the development of antibiotics is struggling to keep pace with the spread of resistant bacteria, the identification of compounds such as analogue 2 could help researchers to win victories in the long fight against bacterial infectious diseases.

Julian G. Hurdle and Aditi Deshpande are in the Center for Infectious and Inflammatory Diseases, Institute of Biosciences and Technology, Texas A\&M Health Science Center, Houston, Texas 77030, USA. e-mails: jhurdle@ibt.tamhsc.edu; adeshpande@medicine.tamhsc.edu

1. Hurdle, J. G., O'Neill, A. J., Chopra, I. \& Lee, R. E. Nature Rev. Microbiol. 9, 62-75 (2011).

2. Kim, W. et al. Nature 556, 103-107 (2018)

3. Costerton, J. W., Stewart, P. S. \& Greenberg, E. P. Science 284, 1318-1322 (1999).

4. Lewis, K. Nature Rev. Microbiol. 5, 48-56 (2007).

5. Dantes, R. et al. JAMA Int. Med. 173, 1970-1978 (2013).

6. Álvarez, R., Vaz, B., Gronemeyer, H. \& de Lera, Á. R. Chem. Rev. 114, 1-125 (2014)

7. Conlon, B. P. et al. Nature 503, 365-370 (2013).

This article was published online on 28 March 2018.

\section{Observations of the birth of crystals}

\section{Different forms of molecular crystals often have distinct properties, which can greatly influence their potential applications. A way of controlling the crystal form of a protein has now been reported. SEE LETTER P.89}

\section{ROBERT G. ALBERSTEIN \& F. AKIF TEZCAN}

$\mathrm{T}$ he second law of thermodynamics dictates that all things tend towards disorder. Yet molecules and other microscopic particles in liquids frequently arrange themselves into perfectly ordered arrays - crystals - without violating this law. Moreover, a given molecule can often arrange itself into more than one type of array, producing different crystal forms known as polymorphs. These polymorphs can have remarkably different properties despite being composed of the same building blocks.
On page 89 , Van Driessche et al. ${ }^{1}$ report experimental observations of protein molecules as they begin to assemble into clusters that then evolve into distinct polymorphs. Their findings bring fresh insight to the important processes of crystal formation and growth, and polymorph selection.

The everyday consequences of crystal polymorphism are perhaps highlighted best by pharmaceutical drugs, most of which are administered as crystalline solids ${ }^{2}$. Polymorphs of drug molecules often exhibit considerable variation in their ease of manufacture, their shelf life and - crucially — their physical and chemical properties, which greatly influence their physiological efficacies ${ }^{2}$. The selection and manufacture of appropriate polymorphs is a major and costly component of the drug-development process, yet processes for polymorph selection are largely conducted on the basis of trial and error, rather than through molecular design.

The development of rational approaches for the design and control of crystal growth, as well as for polymorph selection, requires an understanding of nucleation - the initial stages of crystallization, in which the building blocks begin to form clusters known as nuclei. Unfortunately, there are two main hurdles to capturing and characterizing crystals at birth. First, the nuclei are typically too small to be visualized in $3 \mathrm{D}$ space using most experimental methods, especially when they consist of atoms or small molecules. Second, such nuclei are, by definition, unstable and therefore form only transiently.

To address the first issue, Van Driessche and colleagues used the protein glucose isomerase (GI) as a building block, the box-like shape and nanometre dimensions of which make it relatively easy to identify using a technique called cryo-transmission electron microscopy (cryoTEM). And to overcome the second issue, they 